\title{
SELECTION OF BARLEY (HORDEUM VULGARE) GENOTYPES BY GYT (GENOTYPE $\times$ YIELD $\times$ TRAIT) BIPLOT TECHNIQUE AND ITS COMPARISON WITH GT (GENOTYPE $\times$ TRAIT)
}

\author{
KARAHAN, T. ${ }^{*}-$ AKGÜN, I. ${ }^{2}$ \\ ${ }^{1}$ Field Crops Department, Institute of Natural Sciences, Isparta University of Applied Sciences, \\ Isparta, Turkey \\ ${ }^{2}$ Department of Field Crops, Faculty of Agricultural Sciences and Technologies, Isparta \\ University of Applied Sciences, Isparta, Turkey \\ *Corresponding author \\ e-mail:turan_karahan@hotmail.com \\ (Received $11^{\text {th }}$ Sep 2019; accepted $28^{\text {th }}$ Nov 2019)
}

\begin{abstract}
The selection of genotypes based on multiple traits and multiple environments is very important to improve stable varieties for breeding programs. Since climatic conditions are very variable and unpredictable, they are very effective in the selection of genotypes based on multiple traits in multiple environmental conditions. Thus, the genotype by yield*trait (GYT) biplot approach was used to determine the best barley candidate from 12 barley genotypes based on multiple (four) environment and multiple (six) traits. In this study, the strengths and weaknesses of each genotype was determined by combining yield and other target traits with GYT biplot method and it was compared with GT (genotype*trait). The stability and general adaptability of each genotype showed differences with between GYT and GT biplot techniques. According to the GT biplot method, advanced lines $(3,9,12)$ and Altikat variety were good genotypes, whereas in the GYT biplot method only Altikat variety was the best genotype based on yield ${ }^{\times}$traits combinations. In addition, it was concluded that the best genotype was not fully determined in the GT biplot method, whereas Altikat variety was the best genotype and based on combined traits by GYT biplot. The study showed that GYT biplot is a very good technique, the ideal and stable genotypes can be detected visually with it, and it can be used to define the best candidate based on combining yield and traits selection in breeding programs.
\end{abstract}

Keywords: physiological traits, genotype, Turkey, multiple traits, yield

\section{Introduction}

Barley (Hordeum vulgare L.) is used by animal feed, malt industries, human food and biodiesel industries and it has been the fourth most produced cereal plant after corn, wheat and rice in the world. The production of barley, ranged between 5.4 and 7.4 million tons depending on the growing seasons and it is the most produced after wheat in Turkey (http://www.fao.org/faostat/en/\#data/QC; Kendal et al., 2019; Oral et al., 2019). Therefore, it is very important to develop high yield varieties which are physiologically and morphologically compatible with different environmental conditions.

Barley breeders have been conducting studies in all fields for many years in order to develop high yielding varieties for multi-environments. It is very difficult to develop varieties which are high-yielding in multi-environments. In addition, many ecological and agronomic problems encountered during breeding process, limiting the success of plant breeders and so they have been struggling to develop different models to overcome these problems. The breeder process is confronted with two problems. The 
first is the negative interaction between the genotype and the environmental interaction (GEI) and the second is genotype yield traits interaction (Yan and Frégeau-Reid, 2018).

The breeders have studied for many years the first subjects (genotype $\mathrm{x}$ environment interactions). The candidate and new varieties have been tested under different environmental conditions by many developed methods (GE, GEI, AMMI) in order to characterize their behavior. In the breeder programs, many researchers who work with cereals in different years and environments (Dogan et al., 2016;Kendal and Tekdal, 2016; Kilic, 2014; Mohammadi et al., 2014) reported that the interaction of genotype $\mathrm{x}$ year $\mathrm{x}$ location (GYL) is very important. The second subjects is to develop varieties that can give good results (high efficiency and quality, resistant to diseases, hospitalization, and drought and temperature stress and frost risk) in different environmental conditions. It is very difficult to improve the best varieties in terms of all traits studied in different environments. Therefore, the breeder has the responsibility for identifying if a trait is positively or negatively associated with grain yield, i.e., identifying if grain yield should be multiplied or divided by a specific trait (Mohammadi, 2019). From this, indices are generated by multiplying the grain yield by the magnitudes of traits in question (positive selection) or by dividing the magnitude of traits by the grain yield (negative selection).

The value of crop cultivars for growers increases, when the breeding is done based on multiple traits. For this purpose, a genotype by yield*trait (GYT) biplot approach recently improved by different researchers (Yan and Frégeau-Reid, 2018; Mohammadi, 2019; Kendal, 2019) and this method provide useful information for genotype evaluation on multiple traits. This methodology identifies strengths and weaknesses of each genotype and provides a superiority index (SI) which allows evaluating genotypes in relation to multi-traits.

The aims of this study are to use GYT biplot and identify which traits are associated with grain yield in barley breeding materials to develop new barley cultivars determined in terms of high yielding, agronomic and physiological traits in different environmental conditions.

\section{Materials and methods}

Twelve spring barley genotypes (two checks) were evaluated in two locations in 20132014 and 2014-2015 growing seasons in Turkey. The information on genotypes are presented in Table 1 and the information about locations are presented in Table 2. The locations and trial pictures are given in Appendix as Photos 1, 2 and 3. The standards used in the research are widely cultivated in the region. Therefore, these varieties were selected as standard. Altıkat cultivar is used in the study as control; because this cultivar was released in 2011 in the research area. So, it is very stable among cultivars which used in research on grain yield, because it is regional and majority barley growers prefer the 6 rows cultivar in this area. Sahin 91 cultivar was used in the study as control; because this cultivar is a national cultivar, and it is a facultative type and used in common in the north part of the region, because it is resistant to cold damage which sometimes occur in spring time. The advanced lines used in the research are genotypes generally developed by ICARDA and recommended to the regions with moderate precipitation. These genotypes are suitable for the conditions of Southeastern Anatolia Region since the temperature values are high and average rainfall areas are suggested during the development period. For this reason, detailed studies on these genotypes will be made and registered as appropriate for the region. 
Table 1. The code, name/pedigree, origin, and spike type of barley genotypes

\begin{tabular}{c|c|c|c}
\hline Code & Name of cultivar and pedigree of lines & Origin & Spike type \\
\hline G5 & ŞAHIN 91 (YEA 1553-1/Eskişehir) & GAPIARTC & 2 rows \\
\hline G1 & NK1272/Moroc 9-75/6/ ..SEA01 04-OS.0S-0SD-0SD-0SD-0SD- & AARI & 2 rows \\
\hline G4 & ARUPO/K8755//MORA/3.. & ICARDA & 2 rows \\
\hline G6 & CBSS00M00098S.0S-0SD-0SD-1SD-0SD-0SD-0SD-0SD & & 2 rows \\
\hline G7 & ARUPO/K8755//.. CBSS00M00098S.0S-0SD-0SD-4SD-0SD-0SD- & ICARDA & 2 rows \\
\hline G8 & RECLA 78/SHYRI 2000..CBSS00M00122S.0S-0SD-0SD-4SD- & ICARDA & 2 rows \\
\hline G10 & ALTIKAT (Arta/4/Arta/3/Hml-.. (ICB96-0601-0AP-10AP-0AP) & GAPIARTC & 6 rows \\
\hline G2 & ROBUST//GLORIA-..CBSS00M00027S.0S-0SD-0SD-1SD-0SD-- & ICARDA & 6 rows \\
\hline G3 & CABUYA/JUGL ..CBSS00M00060S.0S-0SD-0SD-01SD-0SD- & ICARDA & 6 rows \\
\hline G9 & CUCAPAH/PUEBLA/.. CBSS00M00206S.0S--0SD-0SD-5SD-0SD- & ICARDA & 6 rows \\
\hline G11 & TAPIR-BAR/PETUNIA 1...CBWS00WM00056S.0S-0SD-0SD-1SD- - ICARDA & 6 rows \\
\hline G12 & UNKONOWN & AARI & 6 rows \\
\hline G: Cultivar, ICARDA: International Center for Agricultural Research in the Dry Areas. GAPIARTC: GAP International \\
Agricultural Research and Training Center: AARI: Aegean Agricultural Research Institute &
\end{tabular}

Table 2. Years, sites, codes and coordinate status of environment

\begin{tabular}{c|c|c|c|c|c|c}
\hline Years & Sites & Altitude $(\mathrm{m})$ & Latitude & Longitude & Soil properties & Average of pers. $(\mathrm{mm})$ \\
\hline $2013-2014$ & Diyarbakır & 612 & $37^{\circ} 55^{\prime} \mathrm{N}$ & $40^{\circ} 14^{\prime} \mathrm{E}$ & $\mathrm{pH}=7.30$ clay-silt & 483.5 \\
$2014-2015$ & Adiyaman & 685 & $37^{\circ} 46^{\prime} \mathrm{N}$ & $38^{\circ} 17^{\prime} \mathrm{E}$ & $\mathrm{pH}=7.50$ clay-silt & 704.3 \\
\hline
\end{tabular}

The trials were carried out in a randomized block design with four replications and sowing density was used 450 seeds per $\mathrm{m}^{-2}$. Plot size was $7.2 \mathrm{~m}^{-2}$ in sowing time $(6 \mathrm{~m}$ long $\times 1.2 \mathrm{~m}$ wide) spacing was $20 \mathrm{~cm}$ and they composed of a total of 6 rows. Sowing of trials were done in November. The fertilizing percentages were used $60 \mathrm{~kg} \mathrm{~N} \mathrm{ha}^{-1}$ and $60 \mathrm{~kg} \mathrm{P} \mathrm{ha}^{-1}$ with planting and $60 \mathrm{~kg} \mathrm{~N} \mathrm{ha}^{-1}$ was used to each plots at tillering time for all plots. Harvesting was done using a Hege 140 harvester in an area of $6 \mathrm{~m}^{2}$ in each plot.

Moreover, grain yield, agronomic traits (plant height, heading time), physiological traits (canopy temperature depression, SPAD chlorophyll meter (Minolta Co. Ltd., Tokyo, Japan) morphological traits (seed number per spike and yield per spike) gathered for each genotype in each plot. The investigated traits were measured as follows.

Plant height: While the plants were yellow, they were obtained by measuring between the bottom of the plants and the peak.

Heading time: It was obtained by calculating between the period of tillering and the period in which 50 percent of the plants in the parcel were spiked.

Canopy temperature depression: Measurements were measured with Rothenbenger precision infrared thermometer when plants were in spike flowering time $(50 \%)$. Measurements were performed by Fisher et al. (1998) and the measurement method and time was measured at an angle of $30^{\circ}$ in the middle of the plot, $50 \mathrm{~cm}$ above the plant height, in the afternoon, in open and windless weather. 
SPAD measurement: While the plants were in the grain filling period, the middle parts of the flag leaf of 10 plants which were randomly selected were determined in SPAD unit by measuring with the SPAD-502 Plus (Minolta SPAD-502, Osaka, Japan) device which is used to measure the chlorophyll amount of the plants. Measurements were measured when plants were in spike flowering time (50\%).

Seed number per spike: The 10 ears randomly taken from each parcel were blended and the values were obtained by counting grains.

Yield per spike: The 10 ears randomly taken from each parcel were blended and the values were obtained by weighing grains.

Sowing and harvesting time: All test plots were sown in the fall (November), which is the optimal sowing time for barley in the trial areas. Harvesting was carried out in July, when the plants were in the harvesting period. The soil properties of the trials area, $\mathrm{pH}=7.30$ and soil is clay-silt. All agronomic application such as weed control and fertilization were practiced uniformly.

\section{Statistical analysis (GT and GYT)}

The data of twelve barley genotypes in multi-location and multi-year trials were analyzed by GT biplot method, as recommended by Yan and Thinker (2005), GYT biplot method, as recommended by Yan and Frégeau-Reid (2018) and Mohammadi (2019). A superiority index (SI) combining all yield-trait integrations were calculated based on the standardized GYT (Yan and Frégeau-Reid 2018). Biplot method was built for all scored traits of genotypes using Genstat 14 release software program. The data were graphically analyzed for interpretation of GT and GYT using the GGE biplot software. Figure $1 A-E$ was produced based on the performance of each genotype for each trait (GT), Figure $2 A-E$ was generated based on the performance of genotypes by yield*traits (GYT).

\section{Results}

\section{The biplot of genotype by trait (GT) and yield trait combination (GYT)}

The mean data of traits across two years in two location of 12 barley genotypes are shown in Table 3. The pairwise correlations among traits of 12 spring barley genotypes are shown in Table 4. These two table data were used to generate a GT biplot Figure 1, although the genotype is compatible with biplot, it represents only $69.01 \%$ of the variation. The genotype by yield*trait (GYT) data for 12 spring barley genotypes across two years in two locations are shown in (Table 5). The data in the GYT table (Table 5) was generated from the GT table (Table 3) and in GYT table. The data in each column consists of a combination of yield-trait. The standardized genotype by yield*trait (GYT) data and superiority index for 12 spring barley genotypes across two years in two locations are shown in Table 6. The genotypes are quite compatible with biplot, they represent $91.58 \%$ of the total variation (PC1 $74.96 \%$, PC2 16.60\%).

Figure $1 A$ visualize the relationships between properties and trait by genotype profiles. Considering the observations on this figure indicated that grain yield was positively correlated with (GY, CTD, SPAD, SNS and YS), while negative corelated with especially quality traits (HT and PH). However, On the other hand the explanations are confirmed by the correlation values in (Table 2). Based on these principles 


$$
\text { - } 1351 \text { - }
$$

described in the GYT biplot technique, the following observations can be made about relationships between yield trait combinations in Figure $2 A$. Considering the observations on this figure indicated that all yield-trait combinations tend to correlate positively with each other because they have yields as a component, as shown by the triangular angles between the vectors (Fig. 2A). This is an important feature of the GYT biplot (Fig. 2) technique, in contrast to the GT biplot (Fig. 1); According to the yieldtrait combinations (below), graphically, it provides the opportunity for genotypes to be ranking in a more meaningful way. However, as shown the strong relationships between the two traits in the GT biplot technique (Fig. 1A), for exam, there is a positive correlation between $\mathrm{GY} * \mathrm{CTD}$ and a negative correlation between $\mathrm{GY} * \mathrm{HT}$ and $\mathrm{GY} * \mathrm{PH}$ (Fig. 1A; Table 3). In GYT biplot technique there is a positive correlation among GY and all traits. Therefore, it is better to select genotypes based on different analysis output.

Table 3. The mean data of tarits across two years in three location of 12 barley genotypes

\begin{tabular}{c|c|c|c|c|c|c|c}
\hline Genotype & GY $\left(\mathbf{k g}_{\left.\mathbf{h} \mathbf{h}^{-1}\right)}\right.$ & SPAD & $\mathbf{C T D}\left({ }^{\circ} \mathbf{C}\right)$ & SNS & YS & HT (date) & PH (cm) \\
\hline G5-Şahin 91 & 4759 & 41.3 & 27.3 & 28.3 & 1.24 & 100 & 108 \\
G1 & 6478 & 42.9 & 28.6 & 33.3 & 1.31 & 95 & 93 \\
G4 & 6464 & 42.3 & 27.2 & 35.8 & 1.51 & 100 & 103 \\
G6 & 6359 & 39.6 & 27.7 & 30.6 & 1.45 & 97 & 93 \\
G7 & 6297 & 40.8 & 28.2 & 39.2 & 1.43 & 100 & 103 \\
G8 & 6076 & 42.1 & 28.4 & 28.3 & 1.27 & 96 & 95 \\
G10-Altıkat & 6978 & 47.1 & 29.4 & 57.5 & 1.93 & 97 & 98 \\
G2 & 5559 & 40.3 & 27.5 & 50.4 & 1.65 & 98 & 108 \\
G3 & 5891 & 40.3 & 28.1 & 51.9 & 1.88 & 101 & 95 \\
G9 & 5660 & 44.7 & 28.2 & 50.0 & 1.94 & 112 & 105 \\
G11 & 5228 & 45.7 & 27.1 & 51.0 & 1.69 & 97 & 108 \\
G12 & 6052 & 41.7 & 28.0 & 58.5 & 2.17 & 97 & 110 \\
Mean & 5983 & 42.4 & 28.0 & 42.9 & 1.62 & 99 & 101 \\
SD & 609.1 & 2.33 & 0.66 & 11.46 & 0.30 & 4.35 & 6.45 \\
LSD (0.05) & 140.5 & 2.2 & 1.8 & 6.8 & 0.3 & 1.2 & 13.3 \\
CV (\%) & 15.17 & 5.18 & 2.78 & 13.8 & 15.5 & 5.80 & 3.95 \\
\hline
\end{tabular}

SD: Standard Deviation, GY: Grain Yield, SPAD: Soil-Plant Analysis Development, CTD: Canopy Temperature Depression, SNS: Seed Number of per Spike, YS: Yield of per Spike, HT: heading time, PH: plant height

Table 4. Pairwaise corelations among traits of 12 spring barley genotypes

\begin{tabular}{c|c|c|c|c|c|c}
\hline & GY & SPAD & CTD & SNS & YS & HT \\
\hline SPAD & 0.16 & & & & & \\
CTD & $0.66^{*}$ & 0.37 & & & & \\
SNS & 0.08 & 0.42 & 0.22 & & & \\
YS & 0.13 & 0.33 & 0.22 & $0.94 * *$ & & \\
HT & -0.28 & 0.12 & -0.02 & 0.17 & 0.29 & \\
PH & $-0.60^{*}$ & 0.11 & -0.51 & 0.40 & 0.35 & 0.26 \\
\hline
\end{tabular}

*Value significant for 0.05 , ** for 0.01 probability level 


$$
\text { - } 1352 \text { - }
$$

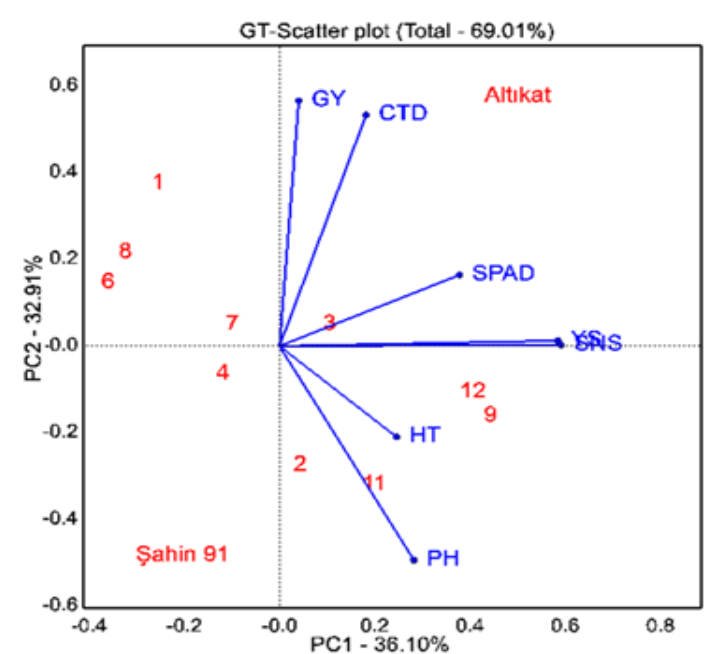

A

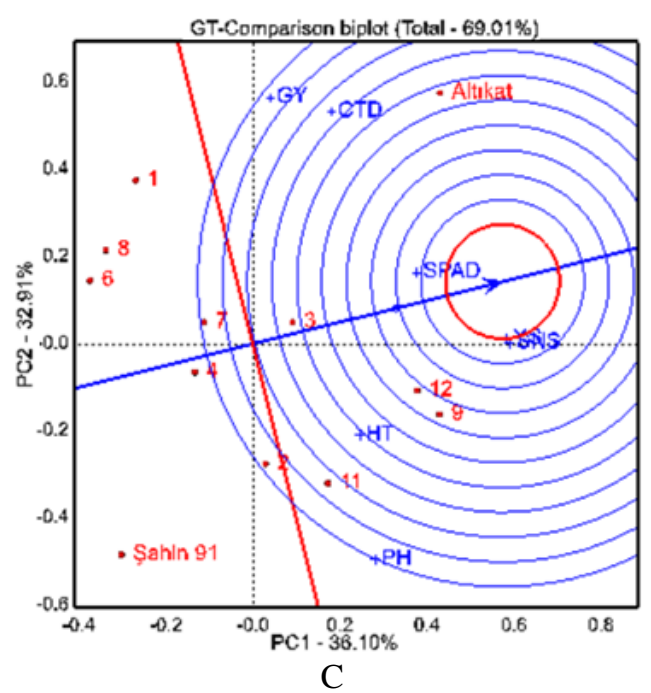

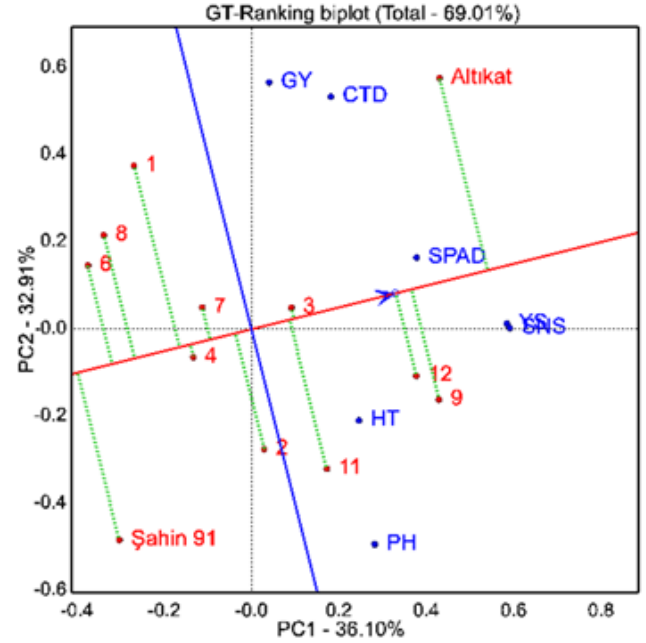

B

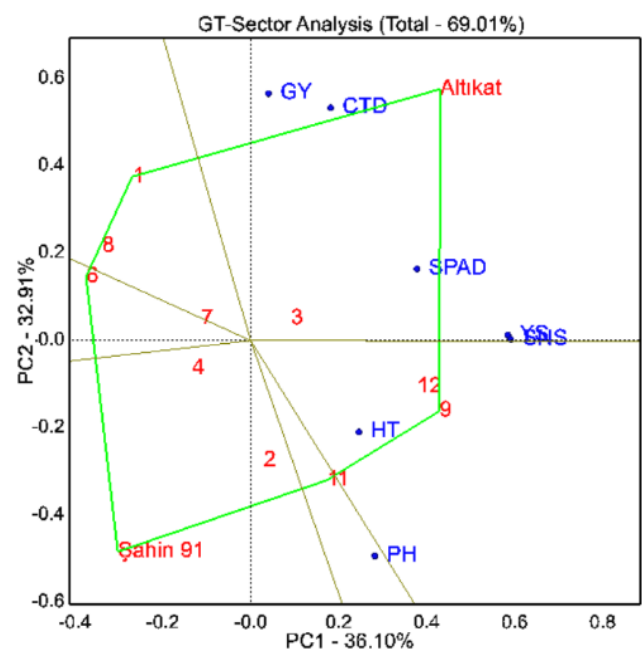

$\mathrm{D}$

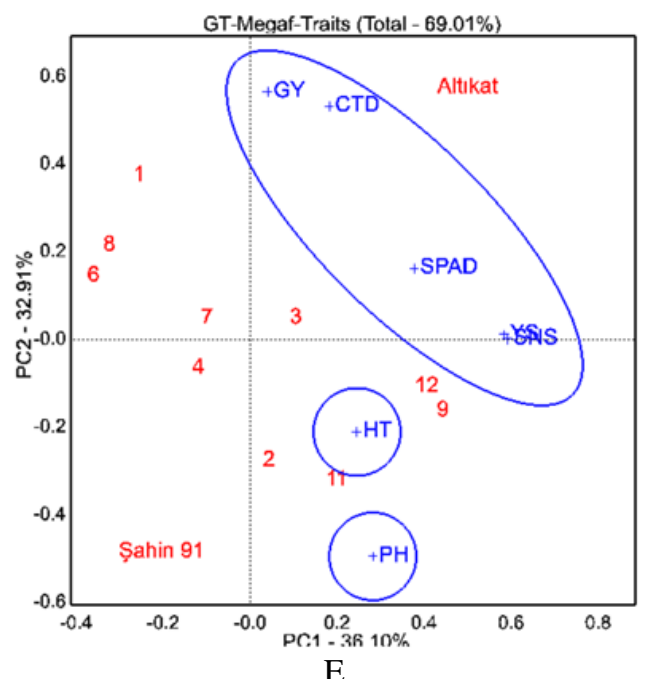

$\mathrm{E}$

Figure 1. This figure was generated based on genotype by trait mean values across two locations and two growing seasons (Tables 3 and 4). $\boldsymbol{A}$ The relationship genotypes and traits. $\boldsymbol{B}$ The stability of genotypes based on traits data. $C$ The comparison of genotypes based on traits data. $\boldsymbol{D}$ Which-won-where/what of GT biplot based on traits data. $\boldsymbol{E}$ The mega-traits based on two seasons and locations data 


$$
\text { - } 1353 \text { - }
$$

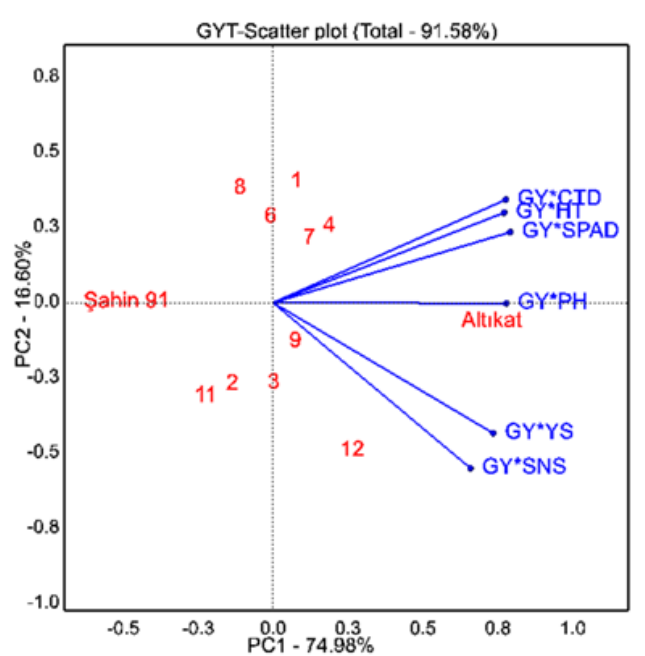

A

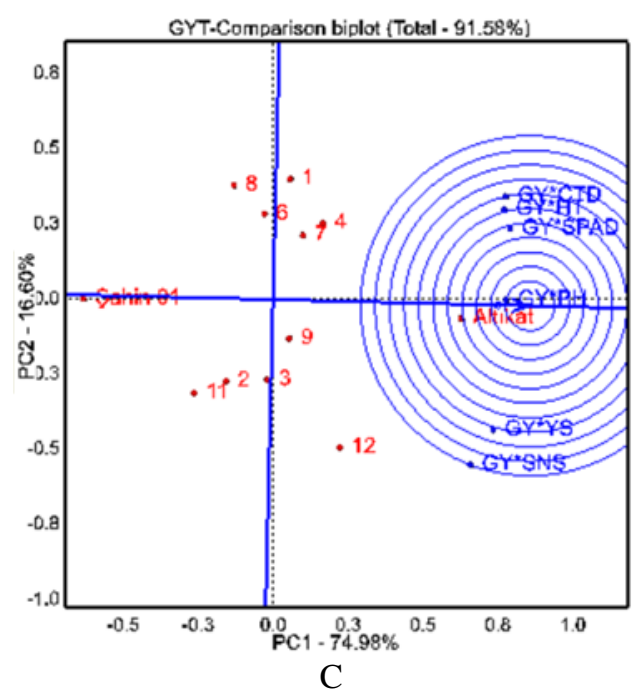

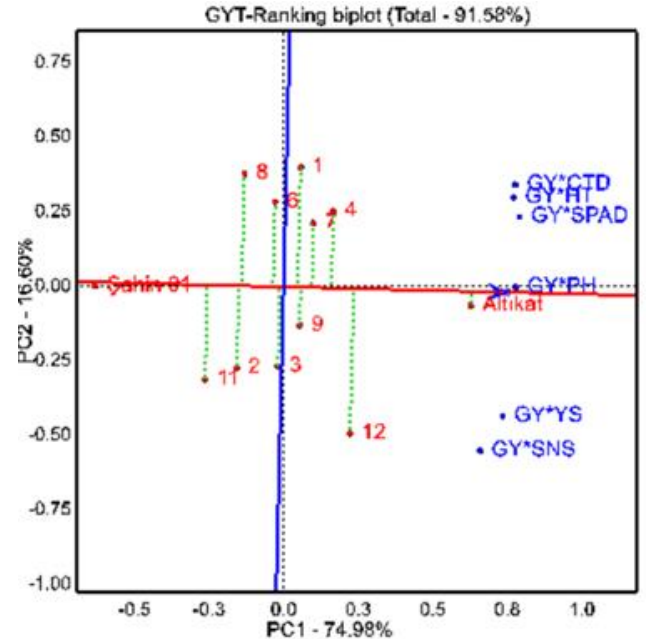

B

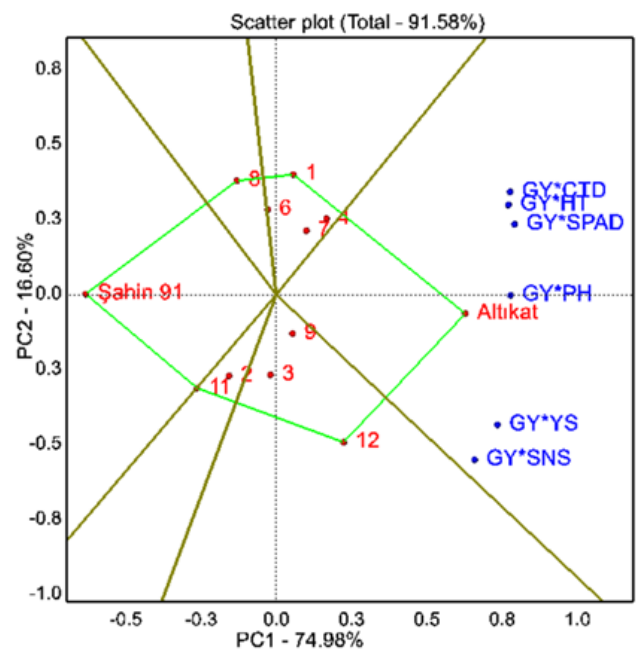

$\mathrm{D}$

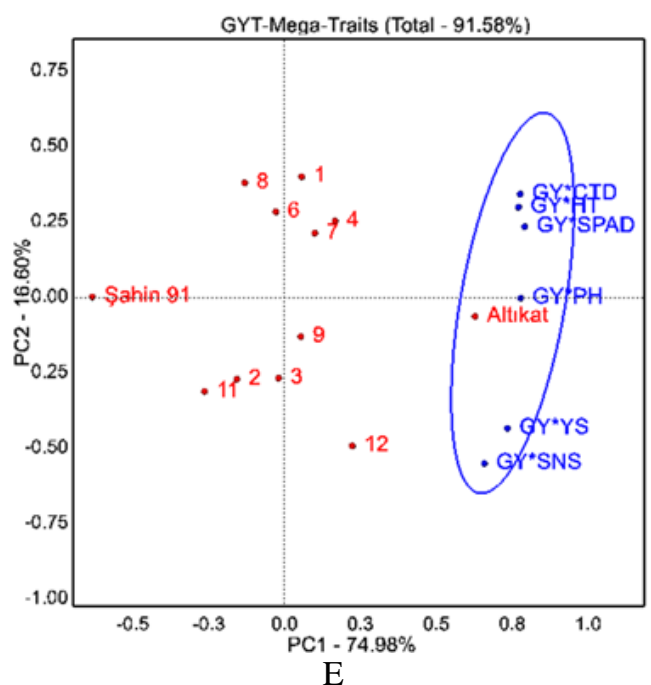

Figure 2. This figure was generated from genotype by yield*trait values across two locations and two growing seasons (Tables 5 and 6 ). $\boldsymbol{A}$ The relationship based on GYT combination data. $\boldsymbol{B}$ The stability based on GYT combination data. $\boldsymbol{C}$ the comparison based on GYT combination data. $\boldsymbol{D}$ Which-won-where/what based on of GYT combination data. $\boldsymbol{E}$ The mega-traits based on GYT combination data 


$$
\text { - } 1354 \text { - }
$$

Table 5. Genotype by yield*trait (GYT) data for 12 spring barley genotypes across two years in two locations

\begin{tabular}{c|c|c|c|c|c|c}
\hline Genotypes & GY*SPAD & GY*CTD & GY*SNS & GY*YS & GY*HT & GY*PH \\
\hline G5-Şahin 91 & 19631 & 12974 & 13447 & 588 & 47768 & 51159 \\
G1 & 27767 & 18495 & 21546 & 849 & 61623 & 59923 \\
G4 & 27326 & 17566 & 23168 & 973 & 64316 & 66256 \\
G6 & 25149 & 17610 & 19474 & 919 & 61600 & 58818 \\
G7 & 25707 & 17773 & 24709 & 900 & 63047 & 64543 \\
G8 & 25566 & 17272 & 17208 & 769 & 58408 & 57724 \\
G-10 Altıkat & 32849 & 20489 & 40096 & 1349 & 67686 & 68035 \\
G2 & 22381 & 15287 & 28013 & 917 & 54406 & 59757 \\
G3 & 23710 & 16531 & 30583 & 1107 & 59569 & 55961 \\
G9 & 25312 & 15985 & 28313 & 1095 & 63104 & 59426 \\
G11 & 23872 & 14148 & 26672 & 881 & 50515 & 56200 \\
G12 & 25207 & 16923 & 35426 & 1313 & 58554 & 66573 \\
Mean & 25373 & 16754 & 25721 & 972 & 59216 & 60364 \\
\hline
\end{tabular}

Table 6. Standardized genotype by yield*trait (GYT) data and superiority index for 12 barley genotypes across two years in two locations

\begin{tabular}{c|c|c|c|c|c|c|c|c}
\hline Genotype & GY*SPAD & GY*CTD & GY*SNS & GY*YS & GY*HT & GY*PH & YLD*SPAD & Mean (SI) \\
\hline G5-Şahin 91 & 0.77 & 0.77 & 0.52 & 0.60 & 0.81 & 0.85 & 0.98 & 0.72 \\
G1 & 1.09 & 1.10 & 0.84 & 0.87 & 1.04 & 0.99 & 0.96 & 1.09 \\
G4 & 1.08 & 1.05 & 0.90 & 1.00 & 1.09 & 1.10 & 0.99 & 1.08 \\
G6 & 0.99 & 1.05 & 0.76 & 0.95 & 1.04 & 0.97 & 1.12 & 0.99 \\
G7 & 1.01 & 1.06 & 0.96 & 0.93 & 1.06 & 1.07 & 1.20 & 1.01 \\
G8 & 1.01 & 1.03 & 0.67 & 0.79 & 0.99 & 0.96 & 1.01 & 1.00 \\
G10-Alt1kat & 1.29 & 1.22 & 1.56 & 1.39 & 1.14 & 1.13 & 0.99 & 1.29 \\
G2 & 0.88 & 0.91 & 1.09 & 0.94 & 0.92 & 0.99 & 1.01 & 0.88 \\
G3 & 0.93 & 0.99 & 1.19 & 1.14 & 1.01 & 0.93 & 0.95 & 0.93 \\
G9 & 1.00 & 0.95 & 1.10 & 1.13 & 1.07 & 0.98 & 0.89 & 0.99 \\
G11 & 0.94 & 0.84 & 1.04 & 0.91 & 0.85 & 0.93 & 0.93 & 0.94 \\
G12 & 0.99 & 1.01 & 1.38 & 1.35 & 0.99 & 1.10 & 0.96 & 0.72 \\
SD & 1.00 & 1.00 & 1.00 & 1.00 & 1.00 & 1.00 & 1.00 & 1.00 \\
\hline
\end{tabular}

Figure $1 B$ visualizes the stability of genotypes based on trait, and in the figure, a horizontal stability axis and a vertical mean axis are created over the average values and the genotypes are evaluated according to these axes. If the genotypes are located under of vertical axis, it means that they are unpreferable if they located above vertical axis, it means that they are preferable genotypes. On the other hand; the genotypes are located near or in the center of the horizontal line it means that these genotypes are stable, and if they move away from the horizontal line, it means that they are unstable (Yan and Rajcan, 2002). Considering Figure 1B with this prediction; 
the advanced line (3) is quite stable because this genotype is located in the center of horizontal axis, and advanced line 12 is stable because this genotype is located near the center of the horizontal axis; Alt1kat variety and advanced line 9,11 etc. are unstable, because they are located far from the center of the horizontal axis. While, Şahin 91(control), advanced line 1 and 8 are unpreferable genotypes because they are located under the vertical axis line, other genotypes (Alt1kat variety, etc), which are located above the axis vertical line, are preferable genotypes based on trait profiles. The effect of GYT to stability and superiority of genotypes are presented in Figure $2 B$. The stability and superiority analysis of GYT indicated that Altikat (control) variety is quite stable because this genotype is located in the center of the horizontal axis, and advanced line 4 is stable because this genotype is located near the center of the horizontal axis; advanced line 12, 11 etc. are unstable, because they are located far from the center of the horizontal axis. While, Şahin 91 (control), advanced line 11 and 8 are unpreferable genotypes because of they located under the vertical axis line, other genotypes (advanced line 1, etc), in which located above the vertical axis line, are preferable genotypes based on trait profiles. The result showed that we have to select different genotypes based on GT and GYT biplot analysis and GYT biplot allows us to make a much clear and more efficient selection in the breeding program.

Figure $1 C$ visualizes the discrimination and representativeness of genotypes based traits, and it provides a representative "ideal center" over the mean values of the properties and offers the opportunity to evaluate genotypes according to their proximity or distance to this center (Yan and Tinker, 2005; Oral, 2018). If the genotypes are located in the center, they are the most ideal, if they are located on the average vertical axis, but far from the center, it means that they are ideal, if they are located below the vertical axis, it means that they are undesirable. Considering Figure $1 C$ with this prediction; advanced line 9 and 12 are more ideal than 3,11 and Alt1kat variety, because it is nearest to the "ideal center", while Şahin 91(control) and advanced lines 1, 6, 8 are located under the vertical axis, and also far from the ideal center, so these genotypes are undesirable. Discrimination and representativeness of genotypes based on GYT combination are presented in Figure $2 C$ and it provides a representative "ideal center" over the mean values of GYT. Considering Figure $2 C$ with this prediction; Alt1kat variety is the most ideal genotype, because it was located nearest to the "ideal center" and advanced lines 12, 9, 4, 7, 6, 1 and 3 are desirable for GYT combination. while Şahin 91 and advanced lines 11, 2, 8 are undesirable genotypes, because these genotypes are located under the mean values of the vertical line. The results of GYT biplot analysis were found to be more clear and stable than GT.

Figure $1 D$ visualizes the polygon of GT biplot (which-won-where/what) based on across season data. The figure divided by thick axis from center figure, and each zone separated by two thick lines is referred to as the "sector" and is indicated by numbers 1 , 2, 3 etc., starting from the lower right part of the graph, and if the genotypes and properties located the same sector it means they are closely related to each other (Yan and Tinker, 2006; Dogan et al., 2016). Considering Figure 1D with this prediction; figure is divided into 6 sectors and different traits are associated with different genotypes in each sector. Genotype 11 is the winner of the sector 1 correlated to $\mathrm{PH}$ trait, advanced line 9 is the winner of the sector 2 located in the same sector with line 12 and correlated to HT. The Alt1kat variety is the winner of the sector 3 located and correlated to GY, CTD, SPAD, SNS and YS. The other genotypes (2, 3, 4, 6, 7, 8, Şahin 91 variety) are located in other sectors and did not correlate with any traits. The GYT 
biplot of sector analysis "which-won-where" can be seen in Figure 2D. The most effective genotype associated with trait profiles in each sector is indicated by a polygon peak. In the sector analysis, the figure was divided into 6 sectors and all combinations were in the same sector. Alt1kat (control) variety located in sector 1 with all combining (GY*CTD, GY*SPAD, GY*SNS, GY*YS, GY*HT, GY*PH). Other genotypes except Alt1kat variety did not correlated with any combinations. The result showed that Alt1kat is the best based on combining of all traits with GY. The result showed that GYT is more effective on the selection of genotypes which is the best based on all traits with grain yield than GT biplot method.

Figure $1 E$ visualizes the group of GT based on across season data and in the figure, the traits and genotypes have relationship, they are located in the center of a circle, it means that there are positive correlation among them (Kendal and Dogan, 2016). Considering Figure $1 E$ in the light of these explanations; features were separated to 3 different mega-traits. The mega-trait 1 were included only (HT) trait, the mega-trait 2 included only (PH) trait, and other traits (GY, CTD, SPAD, SNS, YS) included megatrait 3. The mega- traits based on yield-traits combination across season data are presented in Figure $2 E$, which visualizes to yield-trait combinations, which are in a close relationship, are shown in the same circle. Considering Figure $2 E$ in the light of these explanations; all yield-trait combinations (GY*CTD, GY*SPAD, GY*SNS, $\mathrm{GY}^{*} \mathrm{YS}, \mathrm{GY}^{*} \mathrm{HT}$ and $\left.\mathrm{GY} * \mathrm{PH}\right)$ were located in only one mega-trait and Alt1kat variety was located in the center of this mega-trait. It means that there is correlation among all trait with yield combination. On the other hand, the figure showed that Alt1kat variety is the best genotype based on grain yield with combining traits. The results showed that in a possible selection, the GYT biplot method explained the results more clearly and formally than the GT method.

\section{Discussion}

The GT biplot technique has been used successfully by many researchers for a long time to see the relationship between genotype by trait in different plants, and effective selections were made in breeding programs according to the interaction between genotype by trait. However, GYT biplot technique was designed to complete the deficiencies encountered in the GT biplot technique and to enable more efficient selection of plant breeders. GYT biplot is used to sort genotypes according to their general advantages over yield by trait combinations and to show profiles of traits.

The result showed that the selection of genotypes is based on mega-traits, the use of GYT biplot has more advantages instead of GT biplot in breeding studies. In fact, in barley breeding studies, yield is the only trait that can determine the effectiveness of a genotype alone; other traits (agronomic, morphological and physiological characteristics) are valuable only for the breeders when combined with high yield levels, and these properties alone do not mean anything to growers. For example; a barley genotype is not valuable for breeders if it is resistant to temperature stress and the yield is low. However, if the genotype yield is high, it makes the genotype valuable if it has good agronomic and morphological and physiological characteristics as well as. Therefore, the selection of the best genotypes based on the combination effects of yieldtrait are more meaningful than the effects of individual traits. In the GT biplot technique, a great value (Table 3; Fig. 1B) makes the ATC appearance insignificant in 
some cases (Dehghani et al., 2006; Yan and Tinker, 2006; Kendal and Dogan, 2016; Oral et al., 2018), while in the GYT biplot technique it makes the ATC appearance a meaningful and effective tool (Yan and Frégeau Reid, 2018; Mohammadi, 2019; Kendal, 2019), because it ranks genotypes based on various yield-trait combinations and indicates the strengths and weaknesses of genotypes (Fig. 2B; Table 5). The GT biplot technique was used to construct Figure 1A-E using the data in Table 3, while the GYT biplot technique was used in Figure $2 A-E$ using the data given in Table 5, and genotypes were examined with different graphs according to both techniques. The genotypes examined depend on the superiority index (SI) and yield-trait combination (GYT) and the result of Figure 2 showed that the genotypes can be select easy than GT biplot in Figure 1. On the other hand, in GT biplot it is not clear which is the best genotype that is very stable for all traits, while the Alt1kat is stable and advanced line 4 and 12 for all combination in GYT biplot. Therefore, it was found in this study that GYT biplot technique is a suitable method for determining the most suitable genotype for all properties in barley breeding studies. GYT biplot, in combination with the yield and any trait, is used to measure how the grain yield is combined with that trait in genotypes. When both the grain yield and the values of any trait are low or high, the values will be either low or high and the genotypes will be evaluated accordingly. On the other hand, the GYT biplot technique was developed to determine when the value of a trait of any genotype is low, grain yield is high or vice versa, whether the results are affected from the combination or is there any changing in the ranking of them. As a result, when the values of the traits and the yield values enter the combination, the data changes and the ranking of the genotypes changes. GYT biplot approach has been reported to be a comprehensive and effective method since it classifies genotypes according to their levels in combination with target characteristics and graphically rank the genotypes with their strengths and weaknesses and in different plants (Yan and Frégeau-Reid, 2018).

\section{Conclusion}

The results of this study showed that GYT approach puts too much weight on yield relative to other traits. However, this approach can be used in other crops which is studied based on multi-location, multi-years with multi-traits. The study also verified that there is a potential for simultaneous genetic improvement of the characteristics (SPAD-reading, CTD) in barley. In terms of all traits, the stability of the genotypes and the best genotype is clearly can be seen in the GYT biplot technique (Altıkat), while the best genotypes cannot be seen, because of the GT biplot technique is more complex. Therefore, GYT technique can be used in different plants and traits by researchers for breeding studies.

\section{REFERENCES}

[1] Dehghani, H., Ebadi, A., Yousefi, A. (2006): Biplot analysis of genotype by environment interaction for barley yield in Iran. - Agronomy Journal 98(2): 388-393.

[2] Dogan, Y., Kendal, E., Oral, E. (2016): Identifying of relationship between traits and grain yield in spring barley by GGE Biplot analysis. - Agriculture \& Forestry/Poljoprivreda i Sumarstvo 62(4): 239-252. 
[3] Kendal, E. (2019): Comparing durum wheat cultivars with genotype $\times$ yield $\times$ trait (GYT) and genotype $\times$ trait (GT) by biplot method. - Chilean Journal of Agricultural Research 79(4): 512-522.

[4] Kendal, E, Dogan, Y. (2015): Stability of a candidate and cultivars (Hordeum vulgare L) by GGE Biplot analysis of multi-environment yield trials in spring barley. - Agriculture \& Forestry 61(4): 307-318.

[5] Kendal, E., Karaman, M., Tekdal, S., Doğan, S. (2019): Analysis of promising barley (Hordeum vulgare L.) lines performance by AMMI and GGE biplot in multiple traits and environment. - Applied Ecology and Environmental Research 17(2): 5219-5233.

[6] Kendal, E., \& Tekdal, S. (2016). Application of AMMI model for evaluation spring barley genotypes in multi-environment trials. Bangladesh Journal of Botany, 45(3), 613-620.

[7] Kilic, H. (2014): Additive main effect and multiplicative interactions (AMMI) Analysis of grain yield in barley genotypes across environments, J. - Agr. Sc. 20: 337-344.

[8] Mohammadi, R. (2019): Genotype by yield*trait biplot for genotype evaluation and trait profiles in durum wheat. - Cereal Research Communications 47(3): 541-551.

[9] Mohammadi, R., Haghparast, R., Sadeghzadeh, B., Ahmadi, H., Solimani, K., Amri, A. (2014): Adaptation patterns and yield stability of durum wheat landraces to highland cold rainfed areas of Iran. - Crop Science 54: 944-954.

[10] Oral, E. (2018): Effect of nitrogen fertilization levels on grain yield and yield components in triticale based on AMMI and GGE biplot analysis. - Applied Ecology and Environmental Research 16(4): 4865-4878.

[11] Oral, E., Kendal, E., Dogan, Y. (2018): Selection the best barley genotypes to multi and special environments by AMMI and GGE biplot models. - Fresenius Environmental Bulletin 27(7): 5179-5187.

[12] Oral, E., Kendal, E., Kilic, H., Dogan, Y. (2019): Evolation barley genotypes in multi-environment trials by AMMI model and GGE biplot analysis. - Fresenius Environmental Bulletin 28(4A): 3186-3196.

[13] Solonechnyi, P., Kozachenko, M., Vasko, N., Gudzenko, V., Ishenko, V., Kozelets, G., Vinyukov, A. (2018): AMMI and GGE biplot analysis of yield performance of spring barley (Hordeum vulgare L.) varieties in multi environment trials. - Poljoprivreda i Sumarstvo 64(1): 121-132.

[14] Yan, W., Tinker, N. A. (2005): An integrated biplot analysis system for displaying, interpreting, and exploring genotype $\times$ environment interaction. Crop Science 45(3): 1004-1016.

[15] Yan, W., Tinker, N. A. (2006): Biplot analysis of multi-environment trial data: Principles and applications. - Canadian Journal of Plant Science 86(3): 623-645.

[16] Yan, W., Frégeau-Reid, J. (2018): Genotype by yield * trait (GYT) biplot: a novel approach for genotype selection based on multiple traits. - Sci Rep. 8: 110.

[17] Yan, W., Rajcan, I. R. (2002): Biplot analysis of test sites and trait relations of soybean in Ontario. - Can. J. Plant Sci. 42: 11-20. 


\section{APPENDIX}

Photo 1. The locations of research in Southeast Anatolia of Turkey

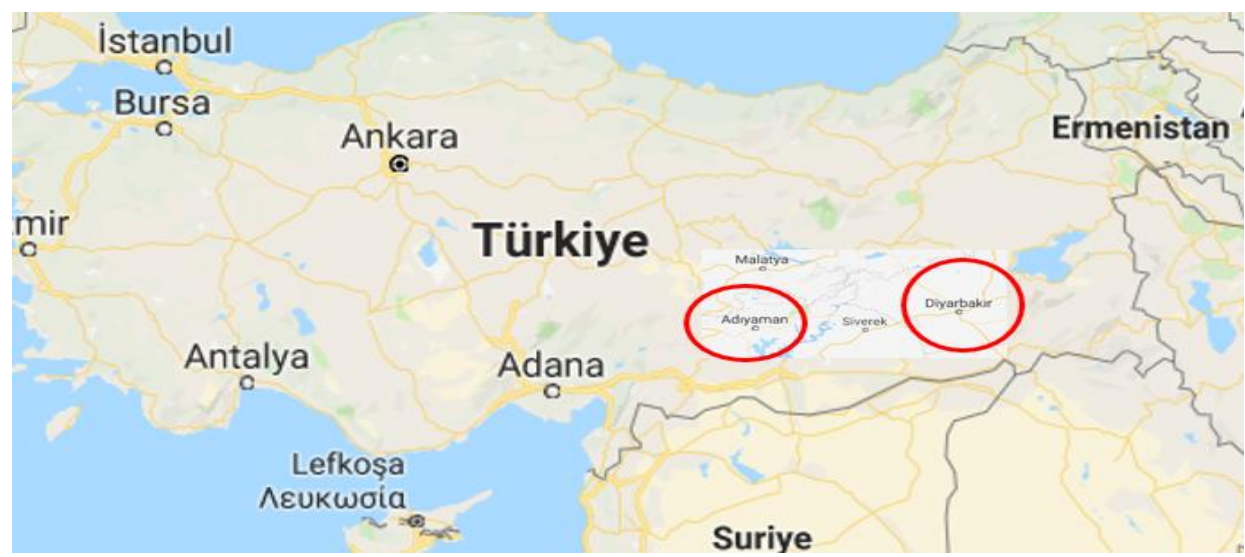

Photo 2. Before and after maturity time of genotypes in the trials in Adyaman

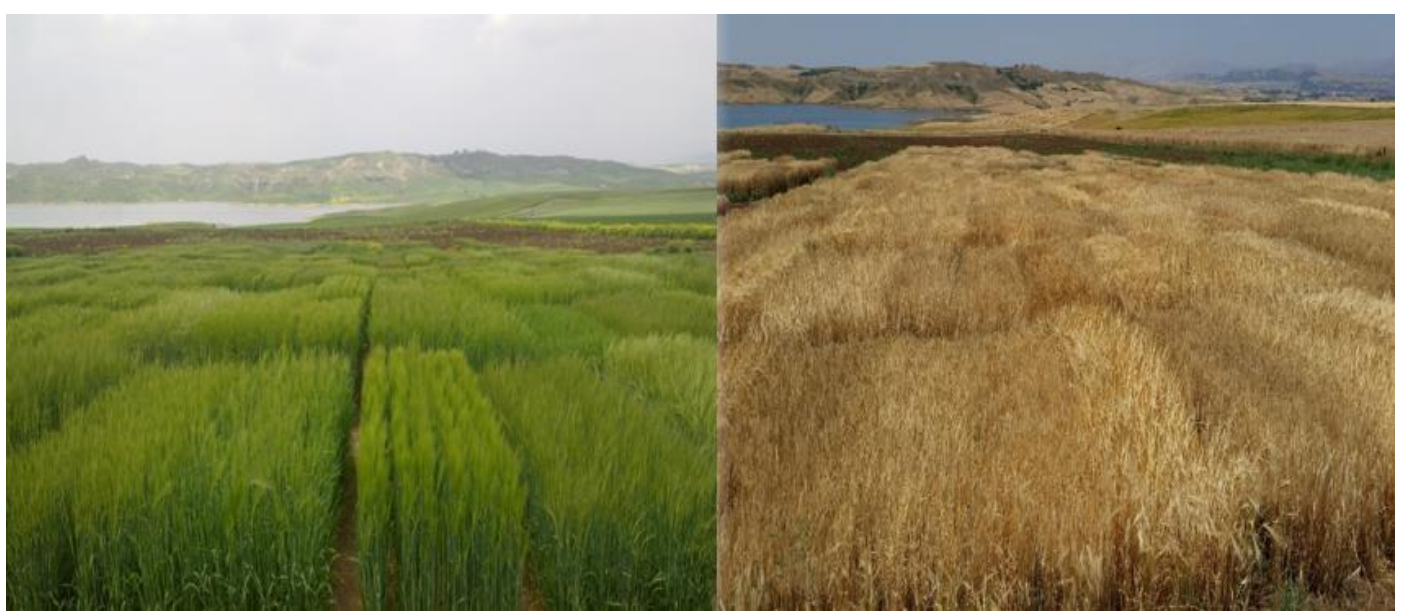

Photo 3. Maturity time of genotypes in the trials in Diyarbakır

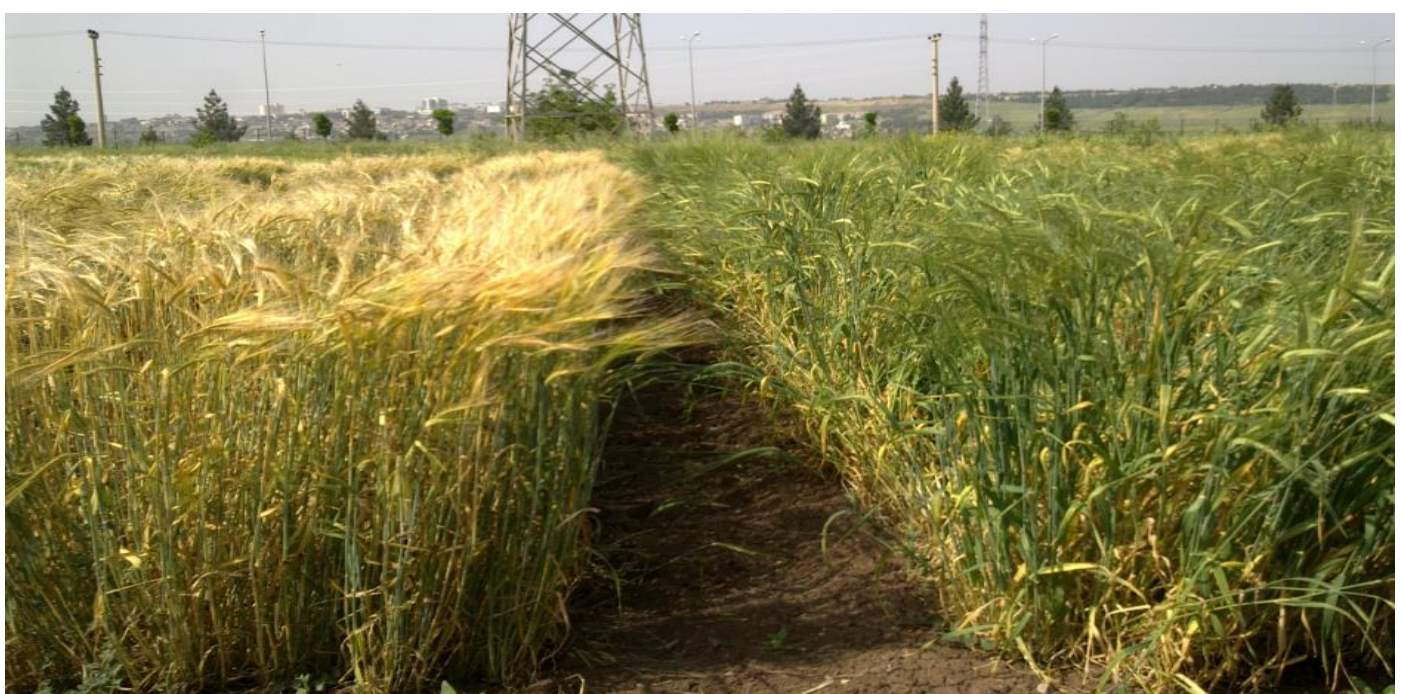

